\section{Calidad de vida relacionada con la salud en personas chilenas con enfermedad pulmonar obstructiva crónica}

\author{
DANIEL COLLADO-MATEO ${ }^{1, \mathrm{a}}$, JOSÉ C. ADSUAR $^{1,2, \mathrm{~b}}$, \\ PEDRO R. OLIVARES ${ }^{3,4, b}$, MIGUEL ÁNGEL GARCÍA-GORDILLO ${ }^{2,5, a}$
}

\section{Health-related quality of life in Chilean patients with chronic obstructive pulmonary disease}

Background: Chronic obstructive pulmonary disease (COPD) has a relevant impact on health-related quality of life (HRQoL). Short Form 6 dimensions (SF-6D) quality of life tool allows researchers to calculate preference-based utilities using data from SF-12 or SF-36 questionnaires. Aim: To provide normative values of SF-6D derived from SF-12 for Chilean patients with COPD. Material and Methods: SF-6D utility index was calculated using data from the 2009/2010 Chilean National Health Survey. Sixty-nine male and 120 female patients with COPD participated in the survey. Data was stratified by gender, age, region, marital status, smoking status, monthly incomes, educational level and area. Results: The mean ( \pm SD) SF-6D utility index for Chilean patients with COPD was $0.65 \pm 0.15$. The scores for men and women were $0.68 \pm 0.15$ and $0.64 \pm 0.15$, respectively. Patients with high incomes and educational level reported higher SF-6D scores. Ceiling effect was not a limitation when SF-6D was used in these Chilean patients. Conclusions: The current study provides normative values of SF-6D derived from SF-12 for Chilean patients with COPD.

(Rev Med Chile 2017; 145: 147-155)

Key words: Lung Diseases; Lung Diseases, Obstructive; Pulmonary Disease, Chronic Obstructive; Quality of life; Reference Values.

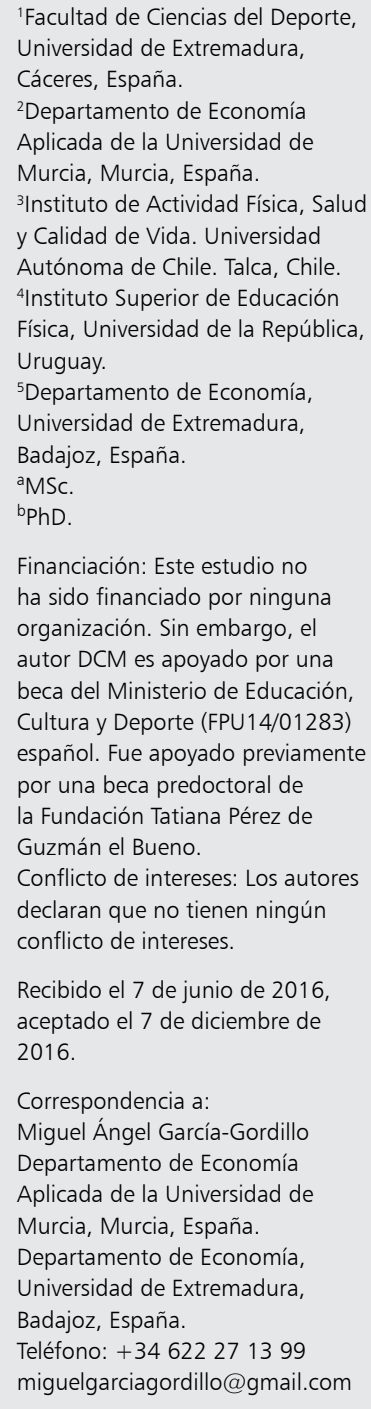

Recibido el 7 de junio de 2016, aceptado el 7 de diciembre de 2016.

Correspondencia a:

Miguel Ángel García-Gordillo

Departamento de Economía

Aplicada de la Universidad de

Murcia, Murcia, España.

Departamento de Economía, Universidad de Extremadura, Badajoz, España.

Teléfono: +34622 271399 miguelgarciagordillo@gmail.com

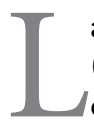

a enfermedad pulmonar obstructiva crónica (EPOC) es un trastorno respiratorio crónico caracterizado por una obstrucción de las vías aéreas, generalmente progresiva $y$, en general, no reversible ${ }^{1}$. El impacto epidemiológico, social y económico de la EPOC es enorme. Se espera que sea la causante del 7,8\% del total de muertes, la cuarta causa de muerte en el mundo en $2030^{2}$ y suponiendo una carga económica sustancial ${ }^{3}$.

Los pacientes que sufren EPOC a menudo tienen una mala calidad de vida relacionada con la salud (CVRS), incluso al inicio de la enfermedad $^{4}$ debido a sus síntomas, a la reducción en la capacidad de realizar actividades de la vida diaria y al uso de medicamentos. La CVRS empeora más en lo físico que en lo mental, y su impacto es más fuerte que la reportada por otros pacientes con enfermedades cardiovasculares o diabetes mellitus ${ }^{5}$.

Muchos cuestionarios están diseñados para evaluar la CVRS. El Short Form-36 (SF-36) ${ }^{6}$ es una de las herramientas más utilizadas. Se compone de 6 dimensiones: funcionamiento físico, limitaciones de rol, funcionamiento social, dolor, salud mental y vitalidad. Consta de 36 preguntas. Esta cantidad de preguntas es superior a otros cuestionarios y podría ser una limitación ${ }^{7}$. El SF- $12^{8}$ 
fue diseñado para hacer frente a esta limitación, reduciendo las preguntas a 12 pero manteniendo las 6 dimensiones del SF-36. El SF-36 tiene otra limitación: no se trata de un cuestionario basado en preferencias poblacionales 9 . En este sentido, el SF-6D ${ }^{10}$ es un cuestionario que consta de 6 preguntas y está basado en preferencias poblacionales, que puede ser utilizado en las evaluaciones económicas.

Hay estudios sobre valores normativos en diferentes países utilizando el SF-6D, como Brasil ${ }^{11}$ o Australia ${ }^{12}$ y en diferentes condiciones, como personas que fueron sometidos a cirugía de fusión lumbar ${ }^{13}$. Sin embargo, no tenemos constancia de que exista ningún estudio sobre valores normativos en chilenos que sufren EPOC. Contar con los valores de referencia de CVRS es un recurso valioso, porque 1) permiten comparaciones entre población enferma y general; 2) pueden ayudar al desarrollo y planificación de la política sanitaria; 3) permiten comparaciones entre países y regiones y 4) facilitan el seguimiento de la CVRS a largo plazo $^{14,15}$. En investigación, los datos normativos permiten la evaluación de la importancia clínica de tratamientos e intervenciones específicas ${ }^{16,17}$ y puede ser un recurso útil para interpretar los resultados reportados por los pacientes ${ }^{18}$.

El objetivo del presente manuscrito es proporcionar los valores normativos de los pacientes chilenos con EPOC.

\section{Material y Métodos}

\section{Diseño y participantes}

Es un estudio transversal que utiliza datos de la Encuesta Nacional de Salud 2009-2010 de Chile. Esta encuesta es propiedad intelectual del Ministerio de Salud de Chile, y está públicamente disponible. Los autores del presente trabajo actual son responsables de todos los resultados del estudio. Estos resultados no son vinculantes para el Ministerio mencionado en ningún sentido.

El marco muestral fue constituido a partir del Censo de Población y Vivienda 2002. El diseño del estudio fue transversal, con una muestra aleatoria de hogares de tipo complejo (estratificada y multietápica por conglomerados) con representatividad nacional, regional y por zona rural/urbana. La población objetivo fueron los adultos de edad mayor o igual a 15 años.
Los análisis se llevaron a cabo para los participantes que respondieron "sí" a la pregunta: "¿Alguna vez le ha dicho un médico que tiene bronquitis crónica, enfisema pulmonar, enfermedad pulmonar obstructiva crónica o EPOC?" (Código Pregunta: r18). Un total de 268 pacientes con EPOC cumplían con tal criterio. Sin embargo, dado que habitualmente, la edad mínima aceptada para la consideración del diagnóstico de EPOC es de 40 años, todos los sujetos con una edad inferior fueron excluidos. Como consecuencia, un total de 189 sujetos (69 varones y 120 mujeres) participaron en la encuesta.

\section{Instrumento SF-6D}

Se utilizó el SF-6D, que es un cuestionario genérico basado en preferencias poblacionales para evaluar la CVRS. Por lo tanto, el SF-6D permite el cálculo de los años de vida ajustados por calidad (AVAC) en los análisis coste-utilidad con los datos de 7 preguntas del SF-12, cuestionario administrado por la Encuesta Nacional de Salud de Chile.

Las 6 dimensiones del SF-6D se calcularon utilizando las siguientes preguntas del SF-12: a) función física (Código: CD3, Su salud actual, ¿lo(a) limita para realizar esfuerzos moderados como mover una mesa, barrer, pasar la aspiradora o caminar más de 1 hora?), b) las limitaciones de rol (Códigos: CD6 y CD7, Durante las últimas 4 semanas, ¿con qué frecuencia ha tenido alguno de los siguientes problemas en su trabajo o en sus actividades cotidianas a causa de su salud física...? ¿Tuvo que dejar de hacer algunas tareas en su trabajo o en sus actividades cotidianas? y $i$ Con qué frecuencia ha tenido alguno de los siguientes problemas en su trabajo o en sus actividades cotidianas a causa de algún problema emocional?), c) función social (Código: CD13, Durante las últimas 4 semanas, ¿con qué frecuencia su salud física o los problemas emocionales han dificultado sus actividades sociales (como por ejemplo visitar amigos o familiares)?), d) dolor (Código: CD9, Durante las últimas 4 semanas, si ha tenido algún dolor, ¿hasta qué punto éste ha interferido con sus tareas normales (incluido el trabajo dentro y fuera de la casa)?), e) salud mental (Código: CD12, ¿Con qué frecuencia se sintió desanimado(a) o deprimido(a)?), y f) vitalidad (Código pregunta: CD11, ¿Con qué frecuencia se sintió con mucha energía?). 
Distintos niveles posibles de respuesta son posible para las 6 dimensiones: función física, 3 niveles; limitaciones de rol, 4 niveles; y resto de dimensiones, 5 niveles. Esto posibilita que 7.500 conjuntos de valores (entre 11111 y 345555$)$ puedan ser definidos como posibles estados de salud.

\section{Análisis estadístico}

La media, DE, mediana, rango intercuartílico (RIC) y efecto techo de la utilidad del SF-6D fueron estimados en el estudio. Las puntuaciones de cada dimensión se utilizaron para definir el estado de salud. Este estado es un número de 6 dígitos, donde el primer dígito corresponde con "funcionamiento físico", el segundo con "limitaciones de rol" y así sucesivamente. En suma, el mejor estado de salud sería "111111" y el peor el "345555". Este número de 6 dígitos se puede convertir en un índice de utilidad que se puede utilizar en los análisis económicos.

El algoritmo utilizado para obtener las utilidades del instrumento fue desarrollado por Brazier y Roberts ${ }^{10}$ con población general de Reino Unido, como en otros estudios ${ }^{19,20}$. La utilidad 1 está asociado al estado de salud 111111 (plena salud) y el valor de utilidad 0,345 está asociado al estado de salud 345555 (peor estado de salud). En cuanto al método de estimación, se utilizó la técnica "Standard Gamble".

La frecuencia del estado de salud "111111" se calculó con el fin de evaluar el efecto techo. Los resultados se estratificaron por género, 6 grupos de edad, 15 regiones, 4 estados civiles, tabaquismo, 5 niveles de ingresos netos mensuales del hogar, 3 niveles educativos y área.

Los datos no siguieron una distribución normal (0,005), así las pruebas U de Mann-Whitney y Kruskal Wallis $\mathrm{H}$ fueron utilizadas para el análisis de los subgrupos. Un p-value 0,05 fue utilizado en todas las pruebas para indicar diferencias estadísticamente significativas. El análisis estadístico se realizó con el programa SPSS v23.

\section{Resultados}

En la Tabla 1, la media $( \pm \mathrm{DE})$ de utilidad fue $0,65( \pm 0,15)$. Los hombres reportaron mejor CVRS que las mujeres, con una utilidad de 0,68 ( \pm $0,15)$ y $0,64( \pm 0,15)$, respectivamente. Respecto a la edad, el grupo de entre 70 y 79 años reportó valores más altos $[0,71( \pm 0,32)]$, mientras que las puntuaciones más bajas se observaron en el grupo de entre 80 y 89 años $[0,59( \pm 0,16)]$. Un tercio de las regiones mostraron una puntuación menor que la media nacional: Antofagasta, Bernardo O'Higgins, Maule, Metropolitana y Los Ríos. Los divorciados/separados reportaron puntuaciones más altas, el resto tenían puntuaciones muy similares. Puntuaciones parecidas se dan también entre fumadores y no fumadores. Las personas con mayor nivel educativo y los ingresos mensuales más altos reportaron puntuaciones más altas. Por último, los residentes en área urbana reportaron mejor estado de salud respecto a los del área rural $\{0,66( \pm 0,15)$ y $0,63( \pm 0,12)$ respectivamente $\}$.

La Tabla 2 compara las puntuaciones diferenciando por género. Los hombres tenían una puntuación más alta en casi todos los grupos de edad, excepto en el grupo de entre 40 y 49 años. En términos generales, los hombres reportaron una mejor CVRS que las mujeres, sin importar el estado civil, el tabaquismo, los ingresos mensuales o el área. Por otro lado, las mujeres con mayor educación mostraron puntuaciones mayores $[0,73$ $( \pm 0,12)$ vs $0,66( \pm 0,13)]$.

La distribución porcentual de cada una de las dimensiones del SF-6D puede verse en la Tabla 3. La dimensión funcionamiento físico tiene $3 \mathrm{op}-$ ciones posibles $(1,2 \circ 3)$, donde las puntuaciones más altas indican un peor estado de salud (al igual que el resto de las dimensiones). Más de $41 \%$ de la muestra total informó que no tuvo problema en esta dimensión. El porcentaje de personas con "ningún problema" en la dimensión limitaciones de rol fue de $20,1 \%$, mientras que el $54,5 \%$ la peor opción posible. Para funcionamiento social, dolor, salud mental, y vitalidad los porcentajes fueron de $34,9 \%, 23,8 \%, 15,3 \%$ y $15,3 \%$, respectivamente. El porcentaje de personas que reportaron problemas en la peor opción posible (máximo) fue de $10,1 \%, 14,8 \%, 6,3 \%$ y $5,3 \%$, respectivamente. En términos generales, la frecuencia de hombres que informaron "ningún problema" era mayor que la frecuencia de la mujer en todas las dimensiones excepto en vitalidad.

La Tabla 4 muestra el efecto techo para toda la población desglosada por los distintos estados de salud. El estado de salud más común fue 111111, lo que significa que $2,1 \%$ de la población estudiada declaró que no tuvo problemas en ninguna dimensión. 
Tabla 1. Características de la muestra objeto de estudio. Valores de referencia del SF-6D

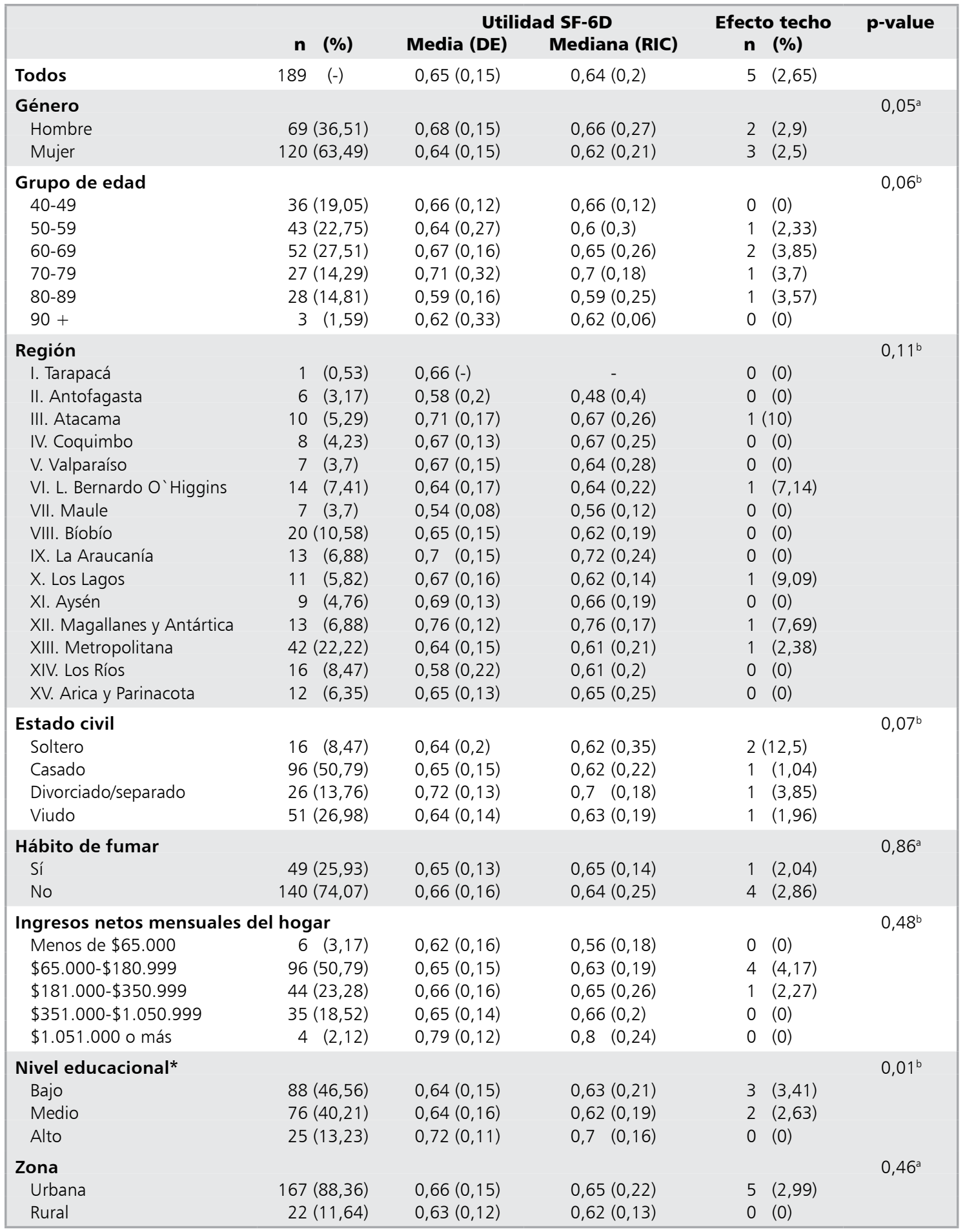

$\$$, Pesos chilenos. *Nivel educacional: Bajo, menos de 8 años de estudios. Medio, entre 8 y 12 años de estudios. Alto: más de 12 años de estudios. ${ }^{a} \mathrm{p}$-value from $\mathrm{U}$ Mann-Whitney test. ${ }^{\mathrm{b}} \mathrm{p}$-value from $\mathrm{H}$ Kruskal-Wallis test. 
Tabla 2. Características de la muestra de estudio. Valores de referencia del SF-6D por género

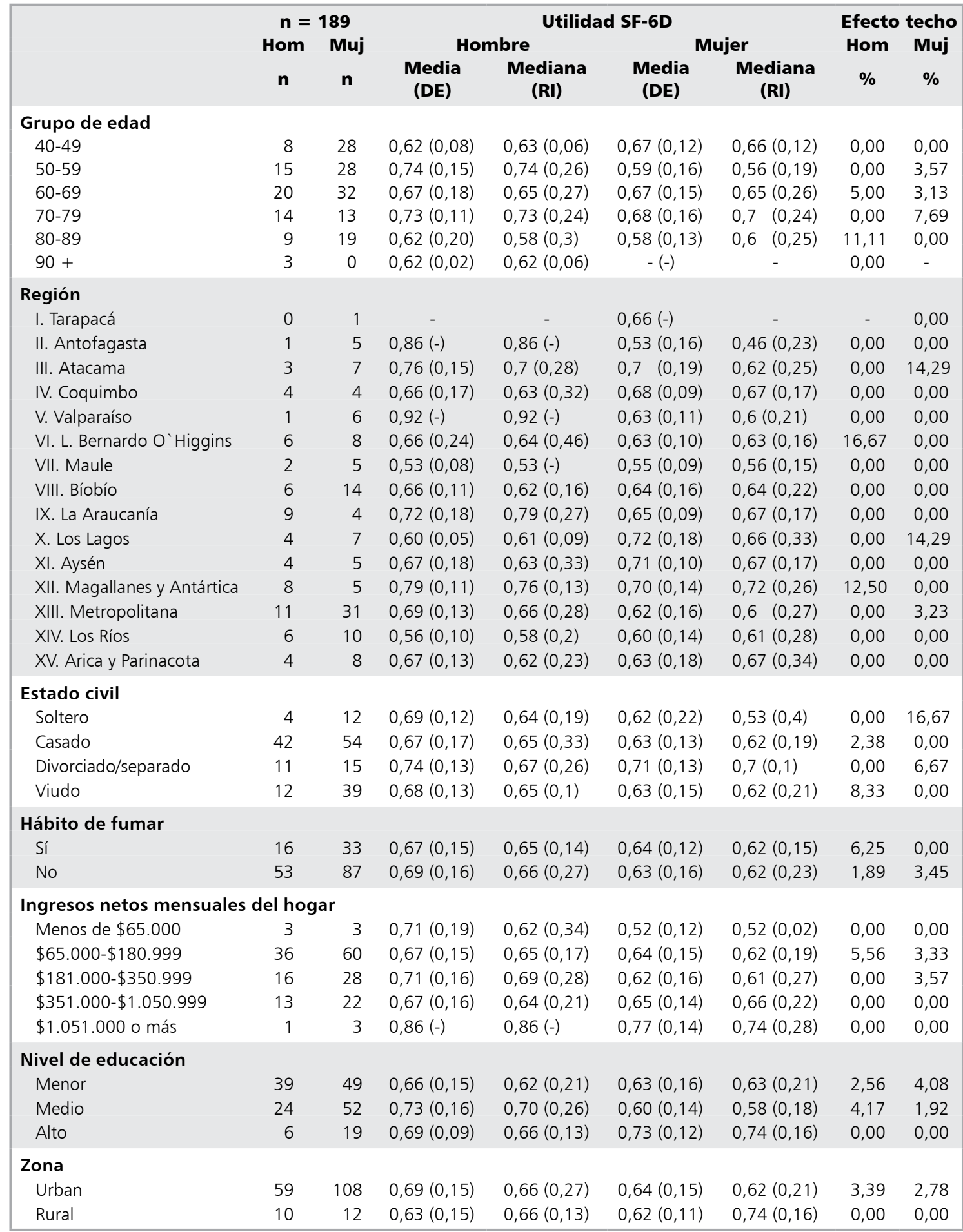

$\$$, Pesos chilenos. *Nivel educacional: Bajo, menos de 8 años de estudios. Medio, entre 8 y 12 años de estudios. Alto: más de 12 años de estudios. 
Tabla 3. Frecuencia porcentual de respuestas (\%) para las 6 dimensiones

\begin{tabular}{|c|c|c|c|c|c|c|c|c|c|c|c|c|c|c|c|c|c|c|}
\hline Nivel & & FF & & & LR & & & FS & & & D & & & SM & & & v & \\
\hline * & Tt & Hom & Muj & Tt & Hom & Muj & $T t$ & Hom & Muj & Tt & Hom & Muj & $\mathrm{Tt}$ & Hom & Muj & $T t$ & Hom & Muj \\
\hline 1 & 41,8 & 46,4 & 39,2 & 20,1 & 26,1 & 16,7 & 34,9 & 44,9 & 29,2 & 23,8 & 26,1 & 22,5 & 15,3 & 23,2 & 10,8 & 15,3 & 11,6 & 17,5 \\
\hline 2 & 30,7 & 26,1 & 33,3 & 16,4 & 15,9 & 16,7 & 21,2 & 20,3 & 21,7 & 22,8 & 29,0 & 19,2 & 20,6 & 21,7 & 20,0 & 27,5 & 27,5 & 27,5 \\
\hline 3 & 27,5 & 27,5 & 27,5 & 9,0 & 7,2 & 10,0 & 21,7 & 14,5 & 25,8 & 15,9 & 11,6 & 18,3 & 39,7 & 42,0 & 38,3 & 33,3 & 42,0 & 28,3 \\
\hline 4 & - & - & - & 54,5 & 50,7 & 56,7 & 12,2 & 14,5 & 10,8 & 22,8 & 20,3 & 24,2 & 18,0 & 11,6 & 21,7 & 18,5 & 14,5 & 20,8 \\
\hline 5 & - & - & - & - & - & - & 10,1 & 5,8 & 12,5 & 14,8 & 13,0 & 15,8 & 6,3 & 1,4 & 9,2 & 5,3 & 4,3 & 5,8 \\
\hline $40-49$ & Tt & Hom & Muj & $T t$ & Hom & Muj & $\mathrm{Tt}$ & Hom & Muj & $\mathrm{Tt}$ & Hom & Muj & $\mathrm{Tt}$ & Hom & Muj & $\mathrm{Tt}$ & Hom & Muj \\
\hline 1 & 58,3 & 62,5 & 57,1 & 22,2 & 0,0 & 28,6 & 36,1 & 50,0 & 32,1 & 25,0 & 0,0 & 32,1 & 0,0 & 0,0 & 0,0 & 5,6 & 0,0 & 7,1 \\
\hline 2 & 27,8 & 12,5 & 32,1 & 13,9 & 25,0 & 10,7 & 22,2 & 12,5 & 25,0 & 36,1 & 50,0 & 32,1 & 19,4 & 25,0 & 17,9 & 30,6 & 25,0 & 32,1 \\
\hline 3 & 13,9 & 25,0 & 10,7 & 16,7 & 12,5 & 17,9 & 30,6 & 25,0 & 32,1 & 16,7 & 12,5 & 17,9 & 58,3 & 50,0 & 60,7 & 41,7 & 37,5 & 42,9 \\
\hline 4 & - & - & - & 47,2 & 62,5 & 42,9 & 5,6 & 12,5 & 3,6 & 13,9 & 25,0 & 10,7 & 19,4 & 25,0 & 17,9 & 19,4 & 25,0 & 17,9 \\
\hline 5 & - & - & - & - & - & - & 5,6 & 0,0 & 7,1 & 8,3 & 12,5 & 7,1 & 2,8 & 0,0 & 3,6 & 2,8 & 2,5 & 0,0 \\
\hline $50-59$ & Tt & Hom & Muj & $T t$ & Hom & Muj & $T t$ & Hom & Muj & $T t$ & Hom & Muj & Tt & Hom & Muj & $T t$ & Hom & Muj \\
\hline 1 & 51,2 & 80,0 & 35,7 & 20,9 & 46,7 & 7,1 & 37,2 & 66,7 & 21,4 & 25,6 & 40,0 & 17,9 & 9,3 & 20,0 & 3,6 & 18,6 & 6,7 & 25,0 \\
\hline 2 & 20,9 & 0,0 & 32,1 & 11,6 & 13,3 & 10,7 & 16,3 & 20,0 & 14,3 & 16,3 & 26,7 & 10,7 & 16,3 & 20,0 & 14,3 & 37,2 & 60,0 & 25,0 \\
\hline 3 & 27,9 & 20,0 & 32,1 & 9,3 & 13,3 & 7,1 & 25,6 & 13,3 & 32,1 & 9,3 & 0,0 & 14,3 & 39,5 & 60,0 & 28,6 & 27,9 & 26,7 & 28,6 \\
\hline 4 & - & - & - & 58,1 & 26,7 & 75,0 & 7,0 & 0,0 & 10,7 & 32,6 & 20,0 & 39,3 & 18,6 & 0,0 & 28,6 & 9,3 & 6,7 & 10,7 \\
\hline 5 & - & - & - & - & - & - & 14,0 & 0,0 & 21,4 & 16,3 & 13,3 & 17,9 & 16,3 & 0,0 & 25,0 & 7,0 & 0,0 & 10,7 \\
\hline $60-69$ & $T t$ & Hom & Muj & $T t$ & Hom & Muj & $T t$ & Hom & Muj & $T t$ & Hom & Muj & $T t$ & Hom & Muj & $T t$ & Hom & Muj \\
\hline 1 & 38,5 & 35,0 & 40,6 & 26,9 & 35,0 & 21,9 & 34,6 & 30,0 & 37,5 & 21,2 & 26,7 & 21,2 & 21,2 & 30,0 & 15,6 & 15,4 & 15,0 & 15,6 \\
\hline 2 & 36,5 & 35,0 & 37,5 & 17,3 & 10,0 & 21,9 & 21,2 & 20,0 & 21,9 & 23,1 & 40,0 & 18,2 & 21,2 & 15,0 & 25,0 & 25,0 & 20,0 & 28,1 \\
\hline 3 & 25,0 & 30,0 & 21,9 & 9,6 & 10,0 & 9,4 & 23,1 & 20,0 & 25,0 & 13,5 & 0,0 & 12,1 & 36,5 & 35,0 & 37,5 & 32,7 & 40,0 & 28,1 \\
\hline 4 & - & - & - & 46,2 & 45,0 & 46,9 & 15,4 & 20,0 & 12,5 & 28,8 & 20,0 & 33,3 & 17,3 & 15,0 & 18,8 & 23,1 & 25,0 & 21,9 \\
\hline 5 & - & - & - & - & - & - & 5,8 & 10,0 & 3,1 & 13,5 & 13,3 & 15,2 & 3,8 & 5,0 & 3,1 & 3,8 & 0,0 & 6,3 \\
\hline $70-79$ & Tt & Hom & Muj & Tt & Hom & Muj & $\mathrm{Tt}$ & Hom & Muj & $T t$ & Hom & Muj & $T t$ & Hom & Muj & Tt & Hom & Muj \\
\hline 1 & 44,4 & 42,9 & 46,2 & 18,5 & 21,4 & 15,4 & 40,7 & 50,0 & 30,8 & 40,7 & 42,9 & 38,5 & 29,6 & 28,6 & 30,8 & 25,9 & 21,4 & 30,8 \\
\hline 2 & 33,3 & 42,9 & 23,1 & 18,5 & 21,4 & 15,4 & 25,9 & 21,4 & 30,8 & 18,5 & 21,4 & 15,4 & 33,3 & 35,7 & 30,8 & 22,2 & 21,4 & 23,1 \\
\hline 3 & 22,2 & 14,3 & 30,8 & 7,4 & 0,0 & 15,4 & 7,4 & 7,1 & 7,7 & 25,9 & 28,6 & 23,1 & 18,5 & 28,6 & 7,7 & 33,3 & 50,0 & 15,4 \\
\hline 4 & - & - & - & 55,6 & 57,1 & 53,8 & 14,8 & 14,3 & 15,4 & 11,1 & 7,1 & 15,4 & 14,8 & 7,1 & 23,1 & 14,8 & 7,1 & 23,1 \\
\hline 5 & - & - & - & - & - & - & 11,1 & 7,1 & 15,4 & 3,7 & 0,0 & 7,7 & 3,7 & 0,0 & 7,7 & 3,7 & 0,0 & 7,7 \\
\hline $80-89$ & Tt & Hom & Muj & Tt & Hom & Muj & $\mathrm{Tt}$ & Hom & Muj & $T t$ & Hom & Muj & $\mathrm{Tt}$ & Hom & Muj & Tt & Hom & Muj \\
\hline 1 & 14,3 & 22,2 & 10,5 & 7,1 & 11,1 & 5,3 & 25,0 & 33,3 & 21,1 & 10,7 & 22,2 & 5,3 & 17,9 & 22,2 & 15,8 & 14,3 & 11,1 & 15,8 \\
\hline 2 & 39,3 & 44,4 & 36,8 & 21,4 & 11,1 & 26,3 & 21,4 & 22,2 & 21,1 & 14,3 & 11,1 & 15,8 & 17,9 & 22,2 & 15,8 & 21,4 & 11,1 & 26,3 \\
\hline 3 & 46,4 & 33,3 & 52,6 & 0,0 & 0,0 & 0,0 & 17,9 & 11,1 & 21,1 & 21,4 & 22,2 & 21,1 & 39,3 & 33,3 & 42,1 & 25,0 & 44,4 & 15,8 \\
\hline 4 & - & - & - & 71,4 & 77,8 & 68,4 & 17,9 & 22,2 & 15,8 & 17,9 & 11,1 & 21,1 & 21,4 & 22,2 & 21,1 & 28,6 & 11,1 & 36,8 \\
\hline 5 & - & - & - & - & - & - & 17,9 & 11,1 & 21,1 & 35,7 & 33,3 & 36,8 & 3,6 & 0,0 & 5,3 & 10,7 & 22,2 & 5,3 \\
\hline $90+$ & $T t$ & Hom & Muj & $T t$ & Hom & Muj & $T t$ & Hom & Muj & $\mathrm{Tt}$ & Hom & Muj & $\mathrm{Tt}$ & Hom & Muj & $\mathrm{Tt}$ & Hom & Muj \\
\hline 1 & 0,0 & 0,0 & 0,0 & 0,0 & 0,0 & 0,0 & 33,3 & 33,3 & 0,0 & 0,0 & 0,0 & 0,0 & 33,3 & 33,3 & 0,0 & 0,0 & 0,0 & 0,0 \\
\hline 2 & 0,0 & 0,0 & 0,0 & 33,3 & 33,3 & 0,0 & 33,3 & 33,3 & 0,0 & 66,7 & 66,7 & 0,0 & 0,0 & 0,0 & 0,0 & 0,0 & 0,0 & 0,0 \\
\hline 3 & 100,0 & 100,0 & 0,0 & 0,0 & 0,0 & 0,0 & 0,0 & 0,0 & 0,0 & 0,0 & 0,0 & 0,0 & 66,7 & 66,7 & 0,0 & 100,0 & 100,0 & 0,0 \\
\hline 4 & - & - & - & 66,7 & 66,7 & 0,0 & 33,3 & 33,3 & 0,0 & 33,3 & 33,3 & 0,0 & 0,0 & 0,0 & 0,0 & 0,0 & 0,0 & 0,0 \\
\hline 5 & - & - & - & - & - & - & 0,0 & 0,0 & 0,0 & 0,0 & 0,0 & 0,0 & 0,0 & 0,0 & 0,0 & 0,0 & 0,0 & 0,0 \\
\hline
\end{tabular}

*Todos los grupos de edad. Tt, Total. Hom, Hombre, Muj, Mujer. FF, Funcionamiento Físico. LR, Llimitaciones de Rol. FS, Funcionamiento Social. D, Dolor. SM, Salud Mental. V, Vitalidad. 
Tabla 4. Patrones de respuestas más frecuentes $(\geq 1 \%)$

\begin{tabular}{|lccc|}
\hline $\begin{array}{l}\text { SF-6D } \\
\text { Estados } \\
\text { salud }\end{array}$ & Frecuencia & $\begin{array}{c}\text { Porcentaje } \\
\text { válido }\end{array}$ & $\begin{array}{c}\text { Porcentaje } \\
\text { acumulado }\end{array}$ \\
\hline 111111 & 4 & 2,1 & 2,1 \\
111112 & 3 & 1,6 & 3,7 \\
111122 & 3 & 1,6 & 5,3 \\
\hline 243333 & 3 & 1,6 & 6,9 \\
\hline 343544 & 3 & 1,6 & 8,5 \\
\hline 111113 & 2 & 1,1 & 9,6 \\
\hline 111133 & 2 & 1,1 & 10,7 \\
142433 & 2 & 1,1 & 11,8 \\
\hline 143233 & 2 & 1,1 & 12,9 \\
\hline 222532 & 2 & 1,1 & 14,0 \\
\hline 243334 & 2 & 1,1 & 15,1 \\
\hline 244233 & 2 & 1,1 & 16,2 \\
\hline 244343 & 2 & 1,1 & 17,3 \\
\hline 344442 & 2 & 1,1 & 18,4 \\
\hline 344444 & 2 & 1,1 & 19,5 \\
\hline 345554 & 2 & 1,1 & 20,6 \\
\hline Otros & 151 & 79,4 & 100 \\
estados & 189 & 100 & \\
\hline & & & \\
\hline
\end{tabular}

\section{Discusión}

Este es el primer estudio que proporciona los valores normativos del SF-6D para la población chilena con EPOC. La utilidad media del SF-6D fue 0,65 . Este valor es inferior a la reportada por los pacientes con EPOC de Portugal $(0,81)^{21} \mathrm{y}$ Suecia $(0,74)^{22}$, pero superior a los pacientes de Canadá $(0,63)^{23}$ y Hong-Kong $(0,63)^{24}$. La puntuación fue menor en mujeres en comparación con los hombres. Este hallazgo es similar a los estudios anteriormente mencionados, aunque la puntuación excepcionalmente alta en la muestra portuguesa podría deberse en parte al 97,2\% de los pacientes fueron varones.

Aunque la muestra está representada por más mujeres que hombres $(63,49 \%$ vs $36,51 \%)$, se presume que puede ser debido al tamaño de la muestra. El estudio encabezado por Amol Dhama$\mathrm{ne}^{25}$ se llevó a cabo con 2.497 sujetos con EPOC y sus porcentajes entre mujeres y hombres era más parejo $(48,8 \%$ vs $51,2 \%)$.

El hábito de fumar supone uno de los factores de riesgos principales para esta población. No obstante, algunos estudios revelan otros factores de riesgos y que, sin embargo, no son recogidos habitualmente en las bases de datos de Encuestas Nacionales de Salud, como la contaminación del aire en lugares de poca ventilación y el componente de la heredabilidad en el desarrollo de EPOC ${ }^{26-29}$.

La educación es importante en la comprensión de la CVRS de los pacientes chilenos con EPOC. Los pacientes con bajo o medio nivel de educación obtuvieron una puntuación de 0,64 mientras que las personas con alto nivel 0,72 . La influencia del nivel educativo es especialmente notable entre las mujeres. Las mujeres chilenas con EPOC y nivel educativo bajo o medio obtuvieron un índice de utilidad de 0,63 y 0,60 respectivamente, el cual es más bajo que el reportado por los hombres $(0,66$ y 0,73 respectivamente). Por otro lado, las mujeres con alto nivel de educación reportaron 0,73 que es mayor que la puntuación reportada por los hombres $(0,69)$. Este hallazgo apoya la relevancia de la educación en la gestión de la EPOC, reduciendo las diferencias entre hombres y mujeres. Estudios previos han informado de que el nivel educativo está estrechamente relacionado con el conocimiento de la propia enfermedad ${ }^{30,31}$ y cómo este conocimiento puede mejorar la capacidad de manejar la enfermedad ${ }^{32}$. Sobre la base de estos resultados, los responsables políticos deberían promover la educación para la salud como una herramienta para la gestión de la enfermedad en pacientes con EPOC. La CVRS en pacientes con EPOC ha sido relacionada con la severidad de la enfermedad en el estudio encabezado por Amol Dhamane ${ }^{25}$. El estudio muestra que aquellas personas que autoreportaron severidad leve tuvieron puntuaciones más altas $(0,75)$. Los de severidad moderada tuvieron 0,70 y 0,67 para aquellos con una severidad mayor.

En cuanto al efecto techo, sólo 2,1\% de los pacientes con EPOC mostró una salud perfecta, es decir, no reportaron ningún problema en ninguna de las dimensiones del SF-6D. De acuerdo con McHorney y Tarlov ${ }^{33}$, se considera que hay efecto techo cuando más de $15 \%$ de la muestra obtiene la puntuación máxima. Por lo tanto, el efecto techo no es una limitación en el SF-6D para los pacientes chilenos con EPOC. 
En relación a las Tablas 1 y 4, puede verse una discrepancia en torno al número y porcentaje del efecto techo para toda la población estudiada. En la Tabla 1 se muestra que 5 sujetos (2,65\%) reportaron salud perfecta, mientras que en la Tabla 4 este valor es de 4 (2,1\%). Esto se debe a que el algoritmo empleado genera un peso a cada dígito de un posible estado de salud, entendiendo diferencias entre cada dimensión evaluada. Por lo tanto, es posible encontrar a un sujeto que reporte plena salud habiendo autoreportado problemas en alguna de las 6 dimensiones del instrumento de medida. En este estudio, una persona autoreportó problemas leves en funcionamiento físico y social (211211) y, sin embargo, obtuvo una utilidad con valor 1 (plena salud).

El presente estudio tiene varias limitaciones. Primero, no hay un algoritmo para calcular la utilidad SF-6D basada en preferencias de la población chilena. En este sentido, se utilizó el algoritmo con las preferencias de las personas del Reino Unido ${ }^{10}$. Las investigaciones futuras deberían centrarse en el desarrollo de un algoritmo basado en las preferencias de la población chilena. Segundo, el tabaquismo es una variable importante en las investigaciones de EPOC por ser uno de los principales factores de riesgo. Sin embargo, los datos no nos permiten hacer diferencias entre los fumadores, no fumadores y exfumadores. Este último grupo se incluye en el grupo de los no fumadores. Por lo tanto, es difícil sacar conclusiones sobre el impacto del tabaco. Otra limitación importante combina el hecho de ser datos autoreportados por los sujetos participantes (atendiendo a la pregunta " $¿$ Alguna vez le ha dicho un médico que tiene bronquitis crónica, enfisema pulmonar, enfermedad pulmonar obstructiva crónica o EPOC?") con la baja sensibilidad y especificidad que subyacen a los síntomas. Algunos estudios epidemiológicos latinoamericanos demuestran que el diagnóstico clínico de EPOC tiene baja sensibilidad y especificidad ${ }^{34,35}$. Ya que, en consecuencia, no puede asumirse con certeza, que la totalidad de los participantes estuviera diagnosticado de EPOC correctamente, sería muy beneficioso para la comunidad científica si en futuras encuestas nacionales de salud, se pudieran precisar tanto la presencia de enfermedad como la severidad de los síntomas.

A pesar de estas limitaciones, el estudio proporciona los valores normativos del SF-6D para los pacientes chilenos con EPOC. El sexo y nivel educativo parecen ser las variables con mayor influencia en las puntuaciones.

Agradecimientos: El autor DCM está recibiendo una beca del Ministerio de España de Educación, Cultura y Deporte (FPU14/01283). $\mathrm{Al}$ autor le fue previamente concedida una beca Predoctoral por parte de la Fundación Tatiana Pérez de Guzmán el Bueno.

\section{Referencias}

1. Decramer M, Janssens W, Miravitlles M. Chronic obstructive pulmonary disease. Lancet 2012; 379: 1341-51.

2. Mathers CD, Loncar D. Projections of global mortality and burden of disease from 2002 to 2030. PLoS medicine 2006; 3: e442.

3. Mannino DM, Higuchi K, Yu TC, Zhou H, Li Y, Tian $\mathrm{H}$, et al. Economic Burden of COPD in the Presence of Comorbidities. Chest 2015; 148: 138-50.

4. Nonato NL, Díaz O, Nascimento OA, Dreyse J, Jardim JR, Lisboa C. Behavior of Quality of Life (SGRQ) in COPD Patients According to BODE Scores. Arch Bronconeumol 2015; 51: 315-21.

5. Janson C, Marks G, Buist S, Gnatiuc L, Gislason T, McBurnie MA, et al. The impact of COPD on health status: findings from the BOLD study. Eur Respir J 2013; 42: 1472-83.

6. Ware JE Jr, Sherbourne CD. The MOS 36-item shortform health survey (SF-36). I. Conceptual framework and item selection. Medical Care 1992; 30: 473-83.

7. Vilagut G, Ferrer M, Rajmil L, Rebollo P, Permanyer-Miralda G, Quintana JM, et al. [The Spanish version of the Short Form 36 Health Survey: a decade of experience and new developments]. Gaceta sanitaria/S.E.S.P.A.S. 2005; 19: 135-50.

8. Ware J Jr, Kosinski M, Keller SD. A 12-Item Short-Form Health Survey: construction of scales and preliminary tests of reliability and validity. Medical Care 1996; 34 : 220-33.

9. Brazier J, Roberts J, Deverill M. The estimation of a preference-based measure of health from the SF-36. J Health Econ 2002; 21: 271-92.

10. Brazier JE, Roberts J. The estimation of a preference-based measure of health from the SF-12. Medical Care 2004; 42: 851-9.

11. Ciconelli RM, Ferraz MB, Kowalski S, da Rocha Castelar Pinheiro G, Sato EI. Brazilian urban population norms derived from the health-related quality of life SF-6D. Qual Life Res 2015; 24: 2559-64.

12. Norman R, Church J, van den Berg B, Goodall S. Aus- 
tralian health-related quality of life population norms derived from the SF-6D. Aust N Z J Public Health 2013; 37: 17-23.

13. Carreon LY, Djurasovic M, Canan CE, Burke LO, Glassman SD. SF-6D values stratified by specific diagnostic indication. Spine 2012; 37: E804-8.

14. Berg B. Sf-6d Population Norms. Health economics 2012; 21: 1508-12.

15. Hawthorne G, Osborne R. Population norms and meaningful differences for the Assessment of Quality of Life (AQoL) measure. Aust N Z J Public Health 2005; 29: 136-42.

16. Kendall PC, Marrs-García A, Nath SR, Sheldrick RC. Normative comparisons for the evaluation of clinical significance. J Consult Clin Psychol 1999; 67: 285-99.

17. Kendall PC GW. Normative comparisons in therapy outcome. Behavioral Assessment 1988; 10 (2): 147-58.

18. Snyder CF, Aaronson NK, Choucair AK, Elliott TE, Greenhalgh J, Halyard MY, et al. Implementing patient-reported outcomes assessment in clinical practice: a review of the options and considerations. Qual Life Res 2012; 21: 1305-14.

19. van Stel HF, Buskens E. Comparison of the SF-6D and the EQ-5D in patients with coronary heart disease. Health Qual Life Outcomes 2006; 4: 20.

20. Kortt MA, Clarke PM. Estimating utility values for health states of overweight and obese individuals using the SF-36. Qual Life Res 2005; 14: 2177-85.

21. Ferreira LN, Ferreira PL, Pereira LN. Comparing the performance of the SF-6D and the EQ-5D in different patient groups. Acta Med Port 2014; 27: 236-45.

22. Szende A, Leidy NK, Stahl E, Svensson K. Estimating health utilities in patients with asthma and COPD: evidence on the performance of EQ-5D and SF-6D. Qual Life Res 2009; 18: 267-72.

23. Lacasse Y, Bernard S, Martin S, Boivin M, Maltais F. Utility Scores In Patients With Oxygen-Dependent COPD: A Case-Control Study. Copd 2015: 1-6.

24. Chen J, Wong CK, McGhee SM, Pang PK, Yu WC. A comparison between the EQ-5D and the SF-6D in patients with chronic obstructive pulmonary disease (COPD). PloS one 2014; 9: e112389.

25. Dhamane AD, Witt EA, Su J. Associations Between COPD Severity and Work Productivity, Health-Related Quality of Life, and Health Care Resource Use:
A Cross-Sectional Analysis of National Survey Data. J Occup Environ Med 2016; 58: e191-7.

26. Lozano R, Naghavi M, Foreman K, Lim S, Shibuya K, Aboyans V, et al. Global and regional mortality from 235 causes of death for 20 age groups in 1990 and 2010: a systematic analysis for the Global Burden of Disease Study 2010. Lancet 2012; 380: 2095-128.

27. McClearn GE, Svartengren M, Pedersen NL, Heller DA, Plomin R. Genetic and environmental influences on pulmonary function in aging Swedish twins. Journal of gerontology 1994; 49: 264-8.

28. Palmer LJ, Knuiman MW, Divitini ML, Burton PR, James AL, Bartholomew HC, et al. Familial aggregation and heritability of adult lung function: results from the Busselton Health Study. Eur Respir J 2001; 17: 696-702.

29. Zhai G, Valdés AM, Cherkas L, Clement G, Strachan D, Spector TD. The interaction of genes and smoking on forced expiratory volume: a classic twin study. Chest 2007; 132: 1772-7.

30. Rani PK, Raman R, Subramani S, Perumal G, Kumaramanickavel G, Sharma T. Knowledge of diabetes and diabetic retinopathy among rural populations in India, and the influence of knowledge of diabetic retinopathy on attitude and practice. Rural Remote Health 2008; 8: 838.

31. Lemes Dos Santos PF, Dos Santos PR, Ferrari GS, Fonseca GA, Ferrari CK. Knowledge of diabetes mellitus: does gender make a difference? Osong Public Health Res Perspect 2014; 5: 199-203.

32. Kim S, Love F, Quistberg DA, Shea JA. Association of health literacy with self-management behavior in patients with diabetes. Diabetes care 2004; 27: 2980-2.

33. McHorney CA, Tarlov AR. Individual-patient monitoring in clinical practice: are available health status surveys adequate? Qual Life Res 1995; 4: 293-307.

34. Tálamo C, de Oca MM, Halbert R, Pérez-Padilla R, Jar$\operatorname{dim}$ JRB, Muino A, et al. Diagnostic labeling of COPD in five Latin American cities. CHEST Journal 2007; 131: 60-7.

35. Herrera AC, de Oca MM, Varela MVL, Aguirre C, Schiavi E, Jardim JR, et al. COPD Underdiagnosis and Misdiagnosis in a High-Risk Primary Care Population in Four Latin American Countries. A Key to Enhance Disease Diagnosis: The PUMA Study. PloS one 2016; 11: e0152266. 